\title{
Multiplicative inequalities for weighted arithmetic and harmonic operator means
}

\author{
Sever S. Dragomir \\ Mathematics, College of Engineering \& Science \\ Victoria University, Melbourne City, Australia \\ email: sever.dragomir@vu.edu.au \\ URL : http: //rgmia.org/dragomir \\ School of Computer Science \& Applied Mathematics, \\ University of the Witwatersrand, \\ Johannesburg, South Africa
}

\begin{abstract}
In this paper we establish some multiplicative inequalities for weighted arithmetic and harmonic operator means under various assumption for the positive invertible operators A, B. Some applications when $A, B$ are bounded above and below by positive constants are given as well.
\end{abstract}

\section{Introduction}

Throughout this paper $A, B$ are positive invertible operators on a complex Hilbert space $(H,\langle\cdot, \cdot\rangle)$. We use the following notations for operators

$$
A \nabla_{v} B:=(1-v) A+v B
$$

the weighted operator arithmetic mean,

$$
A \sharp_{\nu} B:=A^{1 / 2}\left(A^{-1 / 2} B A^{-1 / 2}\right)^{v} A^{1 / 2},
$$

2010 Mathematics Subject Classification: 47A63, 47A30, 15A60, 26D15, 26D10 Key words and phrases: Young's inequality, convex functions, arithmetic mean-harmonic mean inequality, operator means, operator inequalities 
the weighted operator geometric mean and

$$
A !_{\nu} B:=\left((1-v) A^{-1}+v B^{-1}\right)^{-1}
$$

the weighted operator harmonic mean, where $v \in[0,1]$.

When $v=\frac{1}{2}$, we write $A \nabla B, A \sharp B$ and $A$ ! $B$ for brevity, respectively.

The following fundamental inequality between the weighted arithmetic, geometric and harmonic operator means holds

$$
\mathrm{A} !_{\gamma} \mathrm{B} \leq A \sharp_{\nu} \mathrm{B} \leq A \nabla_{\gamma} \mathrm{B}
$$

for any $v \in[0,1]$.

For various recent inequalities between these means we recommend the recent papers [3]-[6], [8]-[12] and the references therein.

The following additive double inequality has been obtained in the recent paper [7]:

$$
v(1-v) \frac{(b-a)^{2}}{\max \{b, a\}} \leq A_{v}(a, b)-H_{v}(a, b) \leq v(1-v) \frac{(b-a)^{2}}{\min \{b, a\}},
$$

for any $a, b>0$ and $v \in[0,1]$, where $A_{v}(a, b)$ and $H_{v}(a, b)$ are the scalar weighted arithmetic mean and harmonic mean, respectively, namely

$$
A_{v}(a, b):=(1-v) a+v b \text { and } H_{v}(a, b):=\frac{a b}{(1-v) b+v a} .
$$

In particular,

$$
\frac{1}{4} \frac{(b-a)^{2}}{\max \{b, a\}} \leq A(a, b)-H(a, b) \leq \frac{1}{4} \frac{(b-a)^{2}}{\min \{b, a\}},
$$

where

$$
A(a, b):=\frac{a+b}{2} \text { and } H(a, b):=\frac{2 a b}{b+a} .
$$

We consider the Kantorovich's constant defined by

$$
K(h):=\frac{(h+1)^{2}}{4 h}, h>0 .
$$

The function $K$ is decreasing on $(0,1)$ and increasing on $[1, \infty), K(h) \geq 1$ for any $h>0$ and $K(h)=K\left(\frac{1}{h}\right)$ for any $h>0$. 
Observe that for any $h>0$

$$
K(h)-1=\frac{(h-1)^{2}}{4 h}=K\left(\frac{1}{h}\right)-1 .
$$

Observe that

$$
K\left(\frac{b}{a}\right)-1=\frac{(b-a)^{2}}{4 a b} \text { for } a, b>0
$$

Since, obviously

$$
a b=\min \{a, b\} \max \{a, b\} \text { for } a, b>0,
$$

then we have the following version of (2):

$$
\begin{aligned}
4 v(1-v) \min \{a, b\}\left[K\left(\frac{b}{a}\right)-1\right] & \leq A_{v}(a, b)-H_{v}(a, b) \\
& \leq 4 v(1-v) \max \{a, b\}\left[K\left(\frac{b}{a}\right)-1\right] .
\end{aligned}
$$

for any $a, b>0$ and $v \in[0,1]$.

For positive invertible operators $A, B$ we define

$$
A \nabla_{\infty} B:=\frac{1}{2}(A+B)+\frac{1}{2} A^{1 / 2}\left|A^{-1 / 2}(B-A) A^{-1 / 2}\right| A^{1 / 2}
$$

and

$$
A \nabla_{-\infty} B:=\frac{1}{2}(A+B)-\frac{1}{2} A^{1 / 2}\left|A^{-1 / 2}(B-A) A^{-1 / 2}\right| A^{1 / 2} .
$$

If we consider the continuous functions $f_{\infty}, f_{-\infty}:[0, \infty) \rightarrow[0, \infty)$ defined by

$$
f_{\infty}(x)=\max \{x, 1\}=\frac{1}{2}(x+1)+\frac{1}{2}|x-1|
$$

and

$$
f_{-\infty}(x)=\max \{x, 1\}=\frac{1}{2}(x+1)-\frac{1}{2}|x-1|,
$$

then, obviously, we have

$$
A \nabla_{ \pm \infty} B=A^{1 / 2} f_{ \pm \infty}\left(A^{-1 / 2} B A^{-1}\right) A^{1 / 2} .
$$

If $A$ and $B$ are commutative, then

$$
A \nabla_{ \pm \infty} B=\frac{1}{2}(A+B) \pm \frac{1}{2}|B-A|=B \nabla_{ \pm \infty} A .
$$

The following additive inequality between the weighted arithmetic and harmonic operator means holds [7]: 
Theorem 1 Let A, B be positive invertible operators and $\mathrm{M}>\mathrm{m}>0$ such that the condition

$$
\mathrm{mA} \leq \mathrm{B} \leq \mathrm{MA}
$$

holds. Then we have

$$
\begin{aligned}
4 v(1-v) g(m, M) A \nabla_{-\infty} B & \leq A \nabla_{v} B-A !_{v} B \\
& \leq 4 v(1-v) G(m, M) A \nabla_{\infty} B
\end{aligned}
$$

where

$$
g(m, M):=\left\{\begin{array}{l}
K(M)-1 \text { if } M<1 \\
0 \text { if } m \leq 1 \leq M \\
K(m)-1 \text { if } 1<m
\end{array}\right.
$$

and

$$
G(m, M):=\left\{\begin{array}{l}
K(m)-1 \text { if } M<1, \\
\max \{K(m), K(M)\}-1 \text { if } m \leq 1 \leq M, \\
K(M)-1 \text { if } 1<m .
\end{array}\right.
$$

In particular,

$$
g(m, M) A \nabla_{-\infty} B \leq A \nabla B-A ! B \leq G(m, M) A \nabla_{\infty} B .
$$

Motivated by the above facts, we establish in this paper some multiplicative inequalities for weighted arithmetic and harmonic operator means under various assumption for the positive invertible operators A, B. Some applications when $A, B$ are bounded above and below by positive constants are given as well.

\section{Multiplicative inequalities}

The following result is of interest in itself:

Lemma 1 For any $\mathrm{a}, \mathrm{b}>0$ and $v \in[0,1]$ we have

$$
v(1-v)\left(1-\frac{\min \{a, b\}}{\max \{a, b\}}\right)^{2} \leq \frac{A_{v}(a, b)}{H_{v}(a, b)}-1 \leq v(1-v)\left(\frac{\max \{a, b\}}{\min \{a, b\}}-1\right)^{2} .
$$

In particular,

$$
\frac{1}{4}\left(1-\frac{\min \{a, b\}}{\max \{a, b\}}\right)^{2} \leq \frac{A(a, b)}{H(a, b)}-1 \leq \frac{1}{4}\left(\frac{\max \{a, b\}}{\min \{a, b\}}-1\right)^{2} .
$$


Proof. We have for any $a, b>0$ and $v \in[0,1]$ that

$$
\begin{aligned}
\frac{A_{v}(a, b)}{H_{v}(a, b)} & =\frac{[(1-v) a+v b][(1-v) b+v a]}{a b} \\
& =\frac{(1-v)^{2} a b+v(1-v) b^{2}+v(1-v) a^{2}+v^{2} a b}{a b} \\
& =\frac{v(1-v)\left(b^{2}+a^{2}\right)+\left(1-2 v+2 v^{2}\right) a b}{a b},
\end{aligned}
$$

which is equivalent with

$$
\frac{A_{v}(a, b)}{H_{v}(a, b)}-1=v(1-v) \frac{(b-a)^{2}}{a b}
$$

for any $a, b>0$ and $v \in[0,1]$.

Since $\min ^{2}\{a, b\} \leq a b \leq \max ^{2}\{a, b\}$ hence

$$
\begin{aligned}
v(1-v) \frac{(b-a)^{2}}{a b} & \leq v(1-v) \frac{(b-a)^{2}}{\min ^{2}\{a, b\}} \\
& =v(1-v)\left(\frac{\max \{a, b\}}{\min \{a, b\}}-1\right)^{2}
\end{aligned}
$$

and

$$
\begin{aligned}
v(1-v) \frac{(b-a)^{2}}{a b} & \geq v(1-v) \frac{(b-a)^{2}}{\max ^{2}\{a, b\}} \\
& =v(1-v)\left(1-\frac{\min \{a, b\}}{\max \{a, b\}}\right)^{2}
\end{aligned}
$$

and by (11) we get the desired result (9).

We observe that the inequality (9) can be written in an equivalent form as

$$
\begin{aligned}
& {\left[v(1-v)\left(1-\frac{\min \{a, b\}}{\max \{a, b\}}\right)^{2}+1\right] H_{v}(a, b)} \\
& \leq A_{v}(a, b) \\
& \leq\left[v(1-v)\left(\frac{\max \{a, b\}}{\min \{a, b\}}-1\right)^{2}+1\right] \mathrm{H}_{v}(a, b)
\end{aligned}
$$


for any $a, b>0$ and $v \in[0,1]$, while (10) as

$$
\begin{aligned}
& {\left[\frac{1}{4}\left(1-\frac{\min \{a, b\}}{\max \{a, b\}}\right)^{2}+1\right] H(a, b)} \\
& \leq A(a, b) \\
& \leq\left[\frac{1}{4}\left(\frac{\max \{a, b\}}{\min \{a, b\}}-1\right)^{2}+1\right] \mathrm{H}(a, b)
\end{aligned}
$$

for any $a, b>0$.

Corollary 1 For any $\mathrm{a}, \mathrm{b} \in[\mathrm{k}, \mathrm{K}] \subset(0, \infty)$ and $v \in[0,1]$ we have

$$
\frac{A_{v}(a, b)}{H_{v}(a, b)}-1 \leq v(1-v)\left(\frac{K}{k}-1\right)^{2} \text {. }
$$

In particular,

$$
\frac{A(a, b)}{H(a, b)}-1 \leq \frac{1}{4}\left(\frac{K}{k}-1\right)^{2} .
$$

We have the following multiplicative inequality between the weighted arithmetic and harmonic operator means:

Theorem 2 Let $\mathrm{A}, \mathrm{B}$ be positive invertible operators and $\mathrm{M}>\mathrm{m}>0$ such that the condition (6) holds. Then we have

$$
\begin{aligned}
& {\left[v(1-v)\left(1-\frac{\min \{M, 1\}}{\max \{m, 1\}}\right)^{2}+1\right] A !_{\nu} B} \\
& \leq A \nabla_{\nu} B \\
& \leq\left[v(1-v)\left(\frac{\max \{M, 1\}}{\min \{m, 1\}}-1\right)^{2}+1\right] A !_{\nu} B
\end{aligned}
$$

for any $v \in[0,1]$.

In particular,

$$
\begin{aligned}
& {\left[\frac{1}{4}\left(1-\frac{\min \{M, 1\}}{\max \{m, 1\}}\right)^{2}+1\right] A ! B} \\
& \leq A \nabla B \\
& \leq\left[\frac{1}{4}\left(\frac{\max \{M, 1\}}{\min \{m, 1\}}-1\right)^{2}+1\right] \text { A } B .
\end{aligned}
$$


Proof. If we write the inequality (12) for $a=1$ and $b=x \in(0, \infty)$ then we have

$$
\begin{aligned}
& {\left[v(1-v)\left(1-\frac{\min \{1, x\}}{\max \{1, x\}}\right)^{2}+1\right]\left(1-v+v x^{-1}\right)^{-1}} \\
& \leq 1-v+v x \\
& \leq\left[v(1-v)\left(\frac{\max \{1, x\}}{\min \{1, x\}}-1\right)^{2}+1\right]\left(1-v+v x^{-1}\right)^{-1} .
\end{aligned}
$$

for any $v \in[0,1]$.

If $x \in[m, M] \subset(0, \infty)$, then $\max \{m, 1\} \leq \max \{x, 1\} \leq \max \{M, 1\}$ and $\min \{\operatorname{m}, 1\} \leq \min \{x, 1\} \leq \min \{M, 1\}$.

We have

$$
\left(\frac{\max \{1, x\}}{\min \{1, x\}}-1\right)^{2} \leq\left(\frac{\max \{M, 1\}}{\min \{m, 1\}}-1\right)^{2}
$$

and

$$
\left(1-\frac{\min \{M, 1\}}{\max \{m, 1\}}\right)^{2} \leq\left(1-\frac{\min \{1, x\}}{\max \{1, x\}}\right)^{2}
$$

for any $x \in[m, M] \subset(0, \infty)$.

Therefore, by (18) we have

$$
\begin{aligned}
& {\left[v(1-v)\left(1-\frac{\min \{M, 1\}}{\max \{m, 1\}}\right)^{2}+1\right]\left(1-v+v x^{-1}\right)^{-1}} \\
& \leq 1-v+v x \\
& \leq\left[v(1-v)\left(\frac{\max \{M, 1\}}{\min \{m, 1\}}-1\right)^{2}+1\right]\left(1-v+v x^{-1}\right)^{-1},
\end{aligned}
$$

for any $x \in[m, M]$ and for any $v \in[0,1]$.

If we use the continuous functional calculus for the positive invertible operator $\mathrm{X}$ with $\mathrm{mI} \leq \mathrm{X} \leq \mathrm{MI}$, then we have from (19) that

$$
\begin{aligned}
& {\left[v(1-v)\left(1-\frac{\min \{M, 1\}}{\max \{m, 1\}}\right)^{2}+1\right]\left((1-v) I+v X^{-1}\right)^{-1}} \\
& \leq(1-v) \mathrm{I}+v X \\
& \leq\left[v(1-v)\left(\frac{\max \{M, 1\}}{\min \{\mathrm{m}, 1\}}-1\right)^{2}+1\right]\left((1-v) \mathrm{I}+v X^{-1}\right)^{-1},
\end{aligned}
$$


for any $v \in[0,1]$.

If we multiply (6) both sides by $A^{-1 / 2}$ we get $M I \geq A^{-1 / 2} B A^{-1 / 2} \geq m I$.

By writing the inequality (20) for $X=A^{-1 / 2} B A^{-1 / 2}$ we obtain

$$
\begin{aligned}
& {\left[v(1-v)\left(1-\frac{\min \{\mathrm{M}, 1\}}{\max \{\mathrm{m}, 1\}}\right)^{2}+1\right]} \\
& \times\left((1-v) \mathrm{I}+v\left(\mathrm{~A}^{-1 / 2} \mathrm{~B} A^{-1 / 2}\right)^{-1}\right)^{-1} \\
& \leq(1-v) \mathrm{I}+v \mathrm{~A}^{-1 / 2} \mathrm{~B} A^{-1 / 2} \\
& \leq\left[v(1-v)\left(\frac{\max \{\mathrm{M}, 1\}}{\min \{\mathrm{m}, 1\}}-1\right)^{2}+1\right] \\
& \times\left((1-v) \mathrm{I}+v\left(\mathrm{~A}^{-1 / 2} \mathrm{BA} A^{-1 / 2}\right)^{-1}\right)^{-1},
\end{aligned}
$$

for any $v \in[0,1]$.

If we multiply the inequality (21) both sides with $A^{1 / 2}$, then we get

$$
\begin{aligned}
& {\left[v(1-v)\left(1-\frac{\min \{\mathrm{M}, 1\}}{\max \{\mathrm{m}, 1\}}\right)^{2}+1\right]} \\
& \times \mathrm{A}^{1 / 2}\left((1-v) \mathrm{I}+v\left(\mathrm{~A}^{-1 / 2} \mathrm{BA}^{-1 / 2}\right)^{-1}\right)^{-1} \mathrm{~A}^{1 / 2} \\
& \leq(1-v) A+v \mathrm{~B} \\
& \leq\left[v(1-v)\left(\frac{\max \{\mathrm{M}, 1\}}{\min \{\mathrm{m}, 1\}}-1\right)^{2}+1\right] \\
& \times \mathrm{A}^{1 / 2}\left((1-v) \mathrm{I}+v\left(\mathrm{~A}^{-1 / 2} \mathrm{~B} A^{-1 / 2}\right)^{-1}\right)^{-1} \mathrm{~A}^{1 / 2},
\end{aligned}
$$

for any $v \in[0,1]$.

Since

$$
\begin{aligned}
& A^{1 / 2}\left((1-v) I+v\left(A^{-1 / 2} B A^{-1 / 2}\right)^{-1}\right)^{-1} A^{1 / 2} \\
& =A^{1 / 2}\left((1-v) I+v A^{1 / 2} B^{-1} A^{1 / 2}\right)^{-1} A^{1 / 2} \\
& =A^{1 / 2}\left(A^{1 / 2}\left((1-v) A^{-1}+v B^{-1}\right) A^{1 / 2}\right)^{-1} A^{1 / 2}
\end{aligned}
$$




$$
\begin{aligned}
& =A^{1 / 2}\left(A^{-1 / 2}\left((1-v) A^{-1}+v B^{-1}\right)^{-1} A^{-1 / 2}\right) A^{1 / 2} \\
& =\left((1-v) A^{-1}+v B^{-1}\right)^{-1}=A !_{\nu} B
\end{aligned}
$$

hence by (22) we get the desired result (16).

We also have:

Theorem 3 Let A, B be positive invertible operators and $\mathrm{M}>\mathrm{m}>0$ such that the condition (6) holds. Then we have

$$
d_{v}(m, M) A !_{\nu} B \leq A \nabla_{\nu} B \leq D_{\nu}(m, M) A !_{\nu} B
$$

for any $v \in[0,1]$, where

$$
\mathrm{d}_{v}(\mathrm{~m}, M):=4\left[\left(v-\frac{1}{2}\right)^{2}+v(1-v) \times\left\{\begin{array}{l}
\mathrm{K}(\mathrm{M}) \text { if } M<1, \\
1 \text { if } \mathrm{m} \leq 1 \leq \mathrm{M}, \\
\mathrm{K}(\mathrm{m}) \text { if } 1<\mathrm{m}
\end{array}\right]\right.
$$

and

$$
\begin{aligned}
& D_{v}(m, M) \\
& :=4\left[\left(v-\frac{1}{2}\right)^{2}+v(1-v) \times\left\{\begin{array}{l}
\mathrm{K}(\mathrm{m}) \text { if } M<1, \\
\max \{\mathrm{K}(\mathrm{m}), \mathrm{K}(\mathrm{M})\} \text { if } \mathrm{m} \leq 1 \leq \mathrm{M}, \\
\mathrm{K}(\mathrm{M}) \text { if } 1<\mathrm{m} .
\end{array}\right.\right.
\end{aligned}
$$

In particular, we have

$$
\mathrm{d}(\mathrm{m}, \mathrm{M}) \mathrm{A} ! \mathrm{B} \leq \mathrm{A} \nabla \mathrm{B} \leq \mathrm{D}(\mathrm{m}, \mathrm{M}) \mathrm{A} ! \mathrm{B}
$$

where

$$
\mathrm{d}(\mathrm{m}, M):=\left\{\begin{array}{l}
\mathrm{K}(\mathrm{M}) \text { if } \mathrm{M}<1 \\
1 \text { if } \mathrm{m} \leq 1 \leq \mathrm{M}, \\
\mathrm{K}(\mathrm{m}) \text { if } 1<\mathrm{m}
\end{array}\right.
$$

and

$$
\mathrm{D}(\mathrm{m}, M):=\left\{\begin{array}{l}
\mathrm{K}(\mathrm{m}) \text { if } \mathrm{M}<1, \\
\max \{\mathrm{K}(\mathrm{m}), \mathrm{K}(M)\} \text { if } \mathrm{m} \leq 1 \leq \mathrm{M}, \\
\mathrm{K}(M) \text { if } 1<\mathrm{m} .
\end{array}\right.
$$

Proof. From (11) we have for any $x \in(0, \infty)$ and for any $v \in[0,1]$ that

$$
\frac{A_{v}(1, x)}{H_{v}(1, x)}-1=v(1-v) \frac{(x-1)^{2}}{x} \text {. }
$$


Since $K(x)-1=\frac{(x-1)^{2}}{4 x}, x>0$, then by $(25)$ we have

$$
\begin{aligned}
\frac{A_{v}(1, x)}{H_{v}(1, x)} & =1+4 v(1-v)[K(x)-1] \\
& =4 v(1-v) \mathrm{K}(x)+4\left(v-\frac{1}{2}\right)^{2} \\
& =4\left[v(1-v) K(x)+\left(v-\frac{1}{2}\right)^{2}\right]
\end{aligned}
$$

or, equivalently,

$$
A_{v}(1, x)=4\left[v(1-v) K(x)+\left(v-\frac{1}{2}\right)^{2}\right] H_{v}(1, x)
$$

for any $x \in(0, \infty)$ and for any $v \in[0,1]$.

From $(26)$ we then have for any $x \in[m, M] \subset(0, \infty)$ that

$$
\begin{aligned}
& 4\left[v(1-v) \min _{x \in[m, M]} K(x)+\left(v-\frac{1}{2}\right)^{2}\right] H_{v}(1, x) \\
& \leq A_{v}(1, x) \leq 4\left[v(1-v) \max _{x \in[m, M]} K(x)+\left(v-\frac{1}{2}\right)^{2}\right] H_{v}(1, x) .
\end{aligned}
$$

Since

$$
\min _{x \in[m, M]} K(x)=\left\{\begin{array}{l}
K(M) \text { if } M<1 \\
1 \text { if } m \leq 1 \leq M \\
K(m) \text { if } 1<m
\end{array}\right.
$$

and

$$
\max _{x \in[m, M]} K(x)=\left\{\begin{array}{l}
K(m) \text { if } M<1, \\
\max \{K(m), K(M)\} \text { if } m \leq 1 \leq M, \\
K(M) \text { if } 1<m,
\end{array}\right.
$$

then by (27) we have

$$
\begin{aligned}
d_{v}(m, M)\left(1-v+v x^{-1}\right)^{-1} & \leq 1-v+v x \\
& \leq D_{v}(m, M)\left(1-v+v x^{-1}\right)^{-1}
\end{aligned}
$$

for any $x \in[m, M]$ and for any $v \in[0,1]$.

By a similar argument to the one from Theorem 2 we deduce the desired operator inequality (23). The details are omitted. 


\section{Some particular cases}

Let $A, B$ positive invertible operators and positive real numbers $m, m^{\prime}, M$, $M^{\prime}$ such that the condition $0<\mathrm{mI} \leq \mathrm{A} \leq \mathrm{m}^{\prime} \mathrm{I}<\mathrm{M}^{\prime} \mathrm{I} \leq \mathrm{B} \leq \mathrm{MI}$ holds.

Put $h:=\frac{M}{m}$ and $h^{\prime}:=\frac{M^{\prime}}{m^{\prime}}$, then we have

$$
A<h^{\prime} A=\frac{M^{\prime}}{m^{\prime}} A \leq B \leq \frac{M}{m} A=h A .
$$

By (16) we get

$$
\begin{aligned}
{\left[v(1-v)\left(\frac{h^{\prime}-1}{h^{\prime}}\right)^{2}+1\right] A !_{\nu} B } & \leq A \nabla_{\gamma} B \\
& \leq\left[v(1-v)(h-1)^{2}+1\right] A !_{\nu} B
\end{aligned}
$$

for any $v \in[0,1]$.

By (23) we get

$$
\begin{aligned}
& 4\left[\left(v-\frac{1}{2}\right)^{2}+v(1-v) \mathrm{K}\left(\mathrm{h}^{\prime}\right)\right] \mathrm{A} !_{\nu} \mathrm{B} \\
& \leq \mathrm{A} \nabla_{\gamma} \mathrm{B} \leq 4\left[\left(v-\frac{1}{2}\right)^{2}+v(1-v) \mathrm{K}(\mathrm{h})\right] \mathrm{A} !_{\nu} \mathrm{B}
\end{aligned}
$$

for any $v \in[0,1]$.

If $0<m I \leq B \leq m^{\prime} I<M^{\prime} I \leq A \leq M I$, then for $h:=\frac{M}{m}$ and $h^{\prime}:=\frac{M^{\prime}}{m^{\prime}}$ we also have

$$
\frac{1}{\mathrm{~h}} \mathrm{~A} \leq \mathrm{B} \leq \frac{1}{\mathrm{~h}^{\prime}} \mathrm{A}<\mathrm{A} \text {. }
$$

Finally, by (16) we get (29) while from (23) we get (30) as well.

\section{References}

[1] S. S. Dragomir, Bounds for the normalized Jensen functional, Bull. Austral. Math. Soc., 74 (3) (2006), 417-478.

[2] S. S. Dragomir, A note on Young's inequality, Preprint RGMIA Res. Rep. Coll., 18 (2015), Art. 126. [Online http://rgmia.org/papers/ v18/v18a126.pdf]. 
[3] S. S. Dragomir, Some new reverses of Young's operator inequality, Preprint RGMIA Res. Rep. Coll., 18 (2015), Art. 130. [http:// rgmia.org/papers/v18/v18a130.pdf] .

[4] S. S. Dragomir, On new refinements and reverses of Young's operator inequality, Preprint RGMIA Res. Rep. Coll., 18 (2015), Art. 135. [http:// rgmia.org/papers/v18/v18a135.pdf] .

[5] S. S. Dragomir, Some inequalities for operator weighted geometric mean, Preprint RGMIA Res. Rep. Coll., 18 (2015), Art. 139. [http://rgmia. org/papers/v18/v18a139.pdf] .

[6] S. S. Dragomir, Some reverses and a refinement of Hölder operator inequality, Preprint RGMIA Res. Rep. Coll. 18 (2015), Art. 147. [http:// rgmia.org/papers/v18/v18a147.pdf] .

[7] S. S. Dragomir, Upper and lower bounds for the difference between the weighted arithmetic and harmonic operator means, Preprint RGMIA Res. Rep. Coll., 19 (2016), Art. [http://rgmia.org/papers/ $\mathrm{v} 19 / \mathrm{v} 19 \mathrm{a} 0 . \mathrm{pdf}]$.

[8] S. Furuichi, Refined Young inequalities with Specht's ratio, J. Egyptian Math. Soc., 20 (2012), 46-49.

[9] S. Furuichi, On refined Young inequalities and reverse inequalities, $J$. Math. Inequal. 5 (2011), 21-31.

[10] W. Liao, J. Wu, J. Zhao, New versions of reverse Young and Heinz mean inequalities with the Kantorovich constant, Taiwanese J. Math., 19 (2015), No. 2, pp. 467-479.

[11] M. Tominaga, Specht's ratio in the Young inequality, Sci. Math. Japon., 55 (2002), 583-588.

[12] G. Zuo, G. Shi, M. Fujii, Refined Young inequality with Kantorovich constant, J. Math. Inequal., 5 (2011), 551-556. 